\title{
Qualitative and Quantitative Analysis of Eating Disorders Related Content on Twitter Social Network
}

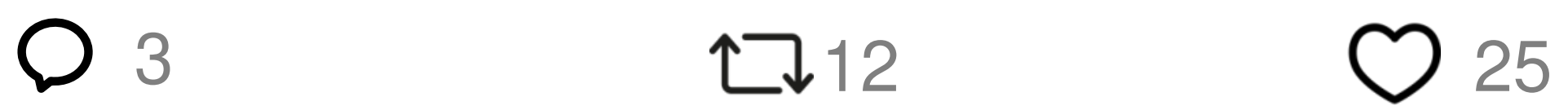

Aymerich, C. ${ }^{1}$, Mancebo, G. ${ }^{1}$, Laría, I. ${ }^{1}$, Urruticoechea, I. ${ }^{1}$, Martínez, A. ${ }^{1}$, Andrés, L. ${ }^{1}$, Paniagua, A. ${ }^{1}$, Blanco, J. ${ }^{1}$, Gómez, D. ${ }^{1}$, Larrañaga, L. ${ }^{1}$, Ruiz-Parra, E. ${ }^{1}$, González-Torres, MA. ${ }^{1}$

${ }^{1}$ Psychiatry Department. Hospital Universitario Basurto. Osakidetza, Bilbao (Spain)

\section{Background and Aim}

Eating disorders (ED) are one of the psychiatric pathologies with the highest mortality and comorbidity rates ${ }^{1}$. Many of those affected never seek professional treatment, turning instead to information and support available through online communities, especially on social networks such as Facebook and Twitter ${ }^{2}$. The data generated online provide a large amount of information that can complement traditional data sources (questionnaires, interviews ...)3.

In all social networks there are ProED communities, which often deny that ED constitute disorders and promote them as a healthy lifestyle alternative. These communities negatively affect the health and the quality of life among those with and without ED, reinforcing thinness and spreading harmful practices for weight loss ${ }^{4,5}$. Unlike other similar platforms, Twitter has not taken any action to moderate this type of content.

The aim of this study was to perform a quantitative and qualitative analysis of the content published on Twitter about anorexia nervosa and bulimia nervosa.

\section{Materials and Methods}

We performed a retrospective, descriptive study. All publicly available messages written in Twitter (tweets) in Spanish language between July 2018 and December 2018, containing the hashtags "\#anorexia", "\#bulimia", "\#proana", "\#promia", and/or "\#anaymia" were collected. The tweets were manually classified and analyzed according to the type of content, its focus and its impact on the social network. A statistical analysis of the messages as a whole and for each different hashtag was performed using IBM SPSSv21 software.

\section{Results}

A total of 1594 tweets were obtained. They were classified into the following categories: $38,4 \%$ informative content; $17,4 \%$ proED content; $16,8 \%$ supportive content; $9,3 \%$ personal testimonies; 9,0\% commercials.

Several hashtags with high sensibility for Pro-ED content where found, i.e. "\#proana" $(\mathrm{N}=167)$, "\#promia" $(\mathrm{N}=119)$ and "\#anaymia" ( $\mathrm{N}=194)$, for which $88,6 \%, 87,4 \%$ and $86,6 \%$ of the tweets containing those hashtags, respectively, were classified as Pro-ED content. These contents are widely and easily accessible for people of all ages.

(Table 1, Graphic 1).

\section{Conclusions}

ProED content in social networks has extensively proved to be harmful not only for ED population, but also for healthy vulnerable population. ${ }^{5}$

Although most of the tweets including the analyzed hashtags belong to the Informative or Pro-Recovery, categories there is a substantial proportion of tweets containing ProED messages. Several highly specific hashtags for this content have been identified: '\#proana', "\#promia", and "anaymia". As of today, there is no moderation in place to restrict these messages to the most vulnerable populations.

Further research should be carried on to identify the real impact of these messages in our setting, both in clinic and general population. Strategies to address such situation should be developed.

\begin{tabular}{|c|c|c|c|c|c|c|c|}
\hline & Informative & ProED & Pro-Recovery & Testimonial & Advertising & Humoristic & Unclassifiable \\
\hline Total $^{*}(\mathrm{~N}=1594)$ & $613(38,5 \%)$ & $278(17,4 \%)$ & $268(16,8 \%)$ & $148(9,3 \%)$ & $144(9,0 \%)$ & $24(1,5 \%)$ & $119(7,5 \%)$ \\
\hline \#proana (N=167) & $6(3,6 \%)$ & $148(88,6 \%)$ & $9(5,4 \%)$ & $3(1,8 \%)$ & $0(0 \%)$ & $0(0 \%)$ & $1(0,6 \%)$ \\
\hline \#promia (N=119) & $4(3,4 \%)$ & $104(87,4 \%)$ & $9(7,6 \%)$ & $2(1,7 \%)$ & $0(0 \%)$ & $0(0 \%)$ & $0(0 \%)$ \\
\hline \#anaymia ( $=194)$ & $12(6,2 \%)$ & $168(86,6 \%)$ & $4(2,1 \%)$ & $1(0,5 \%)$ & $1(0,5 \%)$ & $3(1,6 \%)$ & $5(2,6 \%)$
\end{tabular}

Table 1. Number and percentage of tweets included under each category, divided by hashtags. *Tweets including the following hashtags: \#anorexia, \#bulimia, \#proana, \#promia, \#anaymia. Total $(\mathrm{N}=1594)$
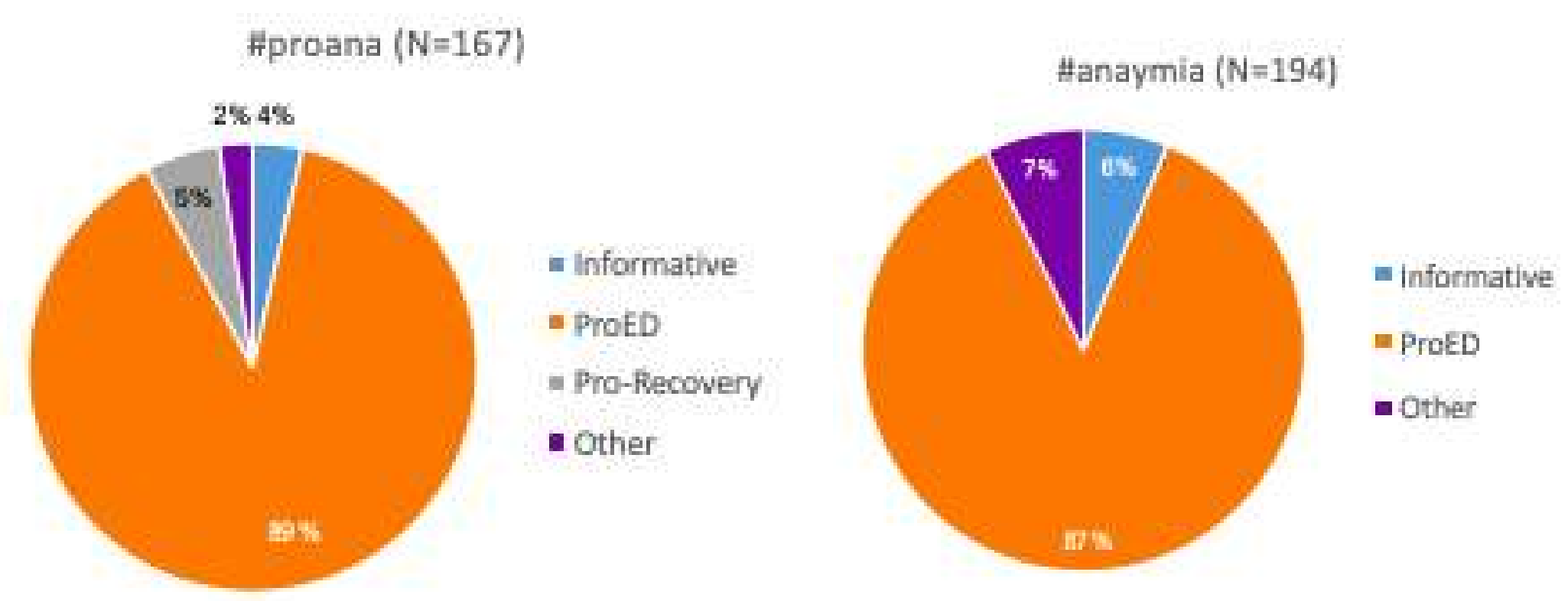

Graphic1. Percentage of tweets included under each category, including the Total sample (left), tweets containing the \#proana hashtag (center) and tweets containing the \#anaymia hashtag (right), two of the hashtags identified as highly specific for ProED content.

Sources: ${ }^{1}$ Arcelus, Mitchell, Wales et al. 2011, ${ }^{2}$ Maloney 2012. ${ }^{3}$ Wang et al. 2019. ${ }^{4}$ Custers 2015. ${ }^{5}$ Branley and Covey 2017. 